\title{
ERGODIC CAPACITY ANALYSIS OF AFWRN WITH IMPERFECT CHANNEL STATE INFORMATION
}

\author{
Dr.M.PremKumar1, and M.Grace Prasana ${ }^{2}$ \\ 1Dept. of ECE, Panimalar Institute of Technology, Poonamalle, Chennai-600 123 \\ 2Dept. of CSE, St.Peters College of Engineering and Technology, Avadi, Chennai-600 054, \\ ermpremkumar@yahoo.co.in, gr_prasana@yahoo.com
}

\section{Abstract-}

This paper deals with Ergodic Capacity analysis of Amplify and Forward Wireless Relay Networks (AFWRN) with Imperfect Channel State Information (ICSI). Ergodic capacity attains prime importance as it provides a limit on the achievable rate in multipath fading channel environments for AFWRN systems. However to analyze ergodic capacity Channel State Information (CSI) is essential and it is practically not available in many scenarios. Hence, CSI needs to be estimated by estimation algorithms such as least squares (LS), minimum mean square error (MMSE) and best linear unbiased estimation (BLUE) algorithms. Here, BLUE algorithm by virtue of its advantages of non-dependence on the complete knowledge of probability density function (PDF), requiring only first and second moments of PDF, and possessing minimal variance characteristics, it is proposed to estimate the CSI of AFWRN system. Using BLUE in AFWRN system ergodic capacity is analysed. The simulation results obtained and presented in this paper provides the tradeoff between perfect CSI and ICSI. Simulation results can be used as a bench mark for analysis of coherent detection in AFWRN systems.

Index Terms_Ergodic Capacity, Amplify and Forward Wireless Relay Networks, Imperfect CSI, BLUE algorithm. (key words)

\section{INTRODUCTION}

Amplify and Forward Wireless Relay Networks (AFWRN)[1]-[3] and [6]-[12] incorporating a number of relay nodes is a prominent research area due to their ability to improve communication coverage, capacity or reliability, spatial diversity of cooperative systems and decrease overall transmit power of wireless networks[1] and[2]. An important tool for the design, appraisal and optimization of AFWRN is ergodic capacity[2].

Ergodic capacity attains the status of an important metric for AFWRN as it yields an information theoretic bound on the achievable rate for reliable communication over fading channels[2] and [3]. Such ergodic capacity can be obtained by taking statistical average over different channel realizations for the instantaneous capacity of an AFWRN. Instantaneous capacity normally requires knowledge of channel state information between the source node to the relay nodes and the relay nodes to the destination node. However, the knowledge of perfect CSI is commonly not available in practical environments but it is usually an assumption made[4] and [5]. Hence, it is required to estimate CSI using estimation algorithms with minimum error variance. Hence, this paper presents ergodic capacity analysis with imperfect channel state information [4] for AFWRN.

Several research works have been reported for analysis of erogdic capacity of AFWRN[6]-[12]. In [5] ergodic capacity and outage capacity performance of a three node wireless relay channel is analyzed in Rayleigh fading scenario were it considered practical constraints on the transmission duplexing at the relaying node and synchronization aspects. Wireless relaying systems with non-regenerative (amplify and forward) relays with variable gain values and fixed gain values were considered with CSI at the receiver in [6] and it also derived an upper bound on ergodic capacity. Ergodic capacity along with the optimal input covariance matrix and amplification coefficients at the relay node is derived with the assumptions of constant amplification coefficients at relay and identical noise power at the relay node and destination node [7]. The performance of dual hop AF 
cooperative networks, with selection cooperation, is investigated in terms of ergodic capacity [8]. A novel Moment Generating Function (MGF) based analytical framework for calculating the ergodic capacities of AF relay networks with limited CSI under three distinct source adaptive transmission policies is presented in [9]. Ergodic channel capacity at lower SNR regions for variable gain and fixed gain amplify and forward relay systems in a generic noise environment, presented in [7] is analyzed and generalized in [10].

Though the works reported so far from [5]-[10] analyzed ergodic capacity of single input single output (SISO) AFWRN with perfect or limited CSI at the destination nodes, and a very few works were reported for imperfect CSI analysis of capacity for AFWRN. In [11], capacity analysis of AFWRN is carried out using channel impulse response coefficients estimated using linear minimum mean square error estimator (LMMSE). Further, a lower bound for the capacity of an AFWRN considering the effect of training and estimation error is also given. This paper derives ergodic capacity analysis of SISO AFWRN with ICSI where ICSI is obtained using BLUE algorithm as given in [12]. BLUE algorithm does not require complete knowledge of PDF, it is based on the first and second moments of PDF and possessing minimal variance characteristics overcomes the drawbacks of LS and MMSE estimators as portrayed in [12].

This paper is written as follows. Section II presents ergodic capacity analysis of SISO AFWRN with ICSI. Section III presents simulation results for SISO AFWRN. Section IV, concludes the paper.

Notations: Vectors and matrices are represented as boldface small and capital letters respectively. The transpose, complex conjugate, Hermitian, inverse of the matrix $\mathbf{C}$ denoted by $\mathbf{C}^{T}, \mathbf{C}^{*}, \mathbf{C}^{H}$ and $\mathbf{C}^{-1}$ respectively. $[\mathbf{C}]_{i j}$ is the $(i, j)^{\text {th }}$ entry of $\mathbf{C} ; \mathbf{I}$ is the identity matrix; $E\{\cdot\}$ denotes statistical expectation, $\operatorname{tr}\{\cdot\}$ denotes trace of a matrix and $C N\left(0, \sigma_{f}^{2}\right)$ represents complex Gaussian noise with zero mean and variance $\sigma_{f}^{2}$.

\section{ERGODIC CAPACITY ANALYSIS OF SISO AFWRN}

The system model for single input single output AFWRN shown in Fig.1 with perfect channel state information at the destination node is represented as

$$
\mathbf{d}_{2}=\mathbf{T} \Delta \mathbf{w}+\mathbf{n}_{d}
$$

where $\mathbf{d}_{2}$ is the $M \times 1$ received signal vector at the destination node, $\mathbf{T}=\left[\begin{array}{llll}\mathbf{P}_{1} \mathbf{s}_{1} & \mathbf{P}_{2} \mathbf{s}_{2} & . & . \\ \mathbf{P}_{M} \mathbf{s}_{M}\end{array}\right]$ with $\mathbf{P}_{i} \quad i=1,2 \ldots . M$ represents the $M \times M$ precoding matrix, $\mathbf{s}_{i}$ is a $M \times 1$ transmitted information symbol vector from the $i^{\text {th }}$ relay node, $\Delta$ is a $M \times M$ matrix of scaling factors, $\mathbf{w}$ is $M \times 1$ overall channel coefficient vector and $\mathbf{n}_{d}$ is $M \times 1$ noise vector at the destination node. In order to derive the ergodic capacity of AFWRN with ICSI (1) is re-written as the $M \times 1$ received signal vector at the destination node with ICSI is represented as

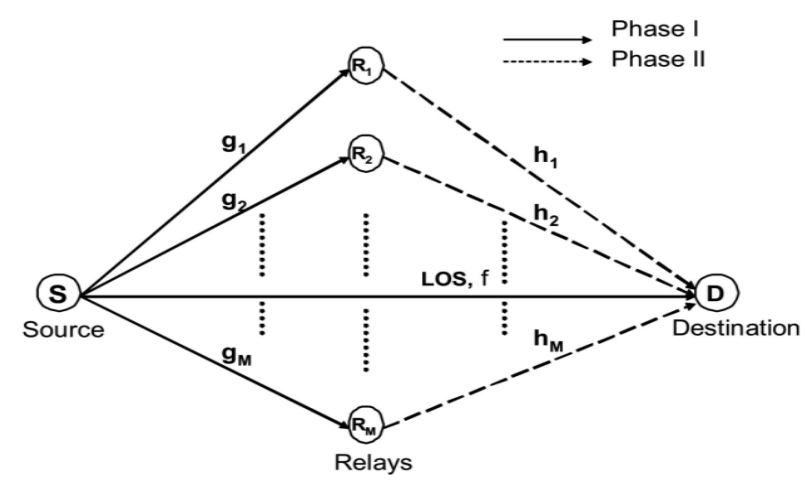

Fig.1 Amplify and Forward Wireless Relay Network

$$
\mathbf{d}_{2 I C S I}=\mathbf{T} \mathbf{\Delta}\left(\hat{\mathbf{w}}_{B L U E}+\mathbf{e}\right)+\mathbf{n}_{d}
$$

where $\hat{\mathbf{w}}_{B L U E}$ is the estimated $M \times 1$ overall channel coefficient vector using BLUE based channel estimation algorithm [12] and $\mathbf{e}$ is a $M \times 1$ error vector which is expressed as

$$
\mathbf{e}=\mathbf{w}-\hat{\mathbf{w}}_{B L U E}
$$

where elements of error vector are complex Gaussian random variable with zero mean and variance $\sigma_{\mathbf{e}}^{2}$. The error variance $\sigma_{\mathbf{e}}^{2}$ is defined as

$$
\sigma_{\mathbf{e}}^{2}=\operatorname{tr}\left\{\left[\mathbf{e} \mathbf{e}^{H}\right]\right\}
$$


In order to determine the error variance of the proposed BLUE estimator for ergodic capacity analysis of SISO AFWRN the estimate of overall channel estimate using the BLUE algorithm is $\hat{\mathbf{w}}_{B L U E}=\left((\mathbf{C} \boldsymbol{\Delta})^{T} \mathbf{R}_{n}^{-1} \mathbf{C} \boldsymbol{\Delta}\right)^{-1}(\mathbf{C} \boldsymbol{\Delta})^{T} \mathbf{R}_{n}^{-1} \mathbf{d}_{2} \quad$ where its derivation can be had from [12]. Using (3) in (4) the error variance is found to be as

$$
\sigma_{\mathbf{e}}^{2}=\operatorname{tr}\left\{(\mathbf{C} \boldsymbol{\Delta})^{-1}\left((\mathbf{C} \boldsymbol{\Delta})^{T}\right)^{H}\left(\left((\mathbf{C} \boldsymbol{\Delta})^{T} \mathbf{R}_{n}^{-1} \mathbf{C} \boldsymbol{\Delta}\right)^{-1}\right)^{H}\right\}
$$

where $\mathbf{C}$ is the $N \times M$ linear transformation matrix, $\boldsymbol{\Delta}$ is $M \times M$ matrix of scaling factors and $\mathbf{R}_{n}$ is the $N \times N$ covariance matrix of overall noise vector at destination node. The capacity of AFWRN with ICSI is given as

$$
C_{I C S I}=\max _{f_{S}(s)}\left[I\left(\mathbf{s} ; \mathbf{d}_{2 I C S I}\right)\right]
$$

where $I\left(\mathbf{s} ; \mathbf{d}_{2 I C S I}\right)$ is the average mutual information between $\mathbf{s}$ and $\mathbf{d}_{2 I C S I}$. The mutual information of AFWRN with ICSI can be written in terms of differential entropy. It is defined as

$$
I\left(\mathbf{s} ; \mathbf{d}_{2 I C S I}\right)=h\left(\mathbf{d}_{2 I C S I}\right)-h\left(\mathbf{d}_{2 I C S I} / \mathbf{s}\right)
$$

where $h\left(\mathbf{d}_{2 I C S I}\right)$ is the differential entropy of the received signal vector with ICSI and $h\left(\mathbf{d}_{2 I C S I} / \mathbf{s}\right)$ is the conditional differential entropy or noise entropy vector with ICSI. As the received signal vector $\mathbf{d}_{2 I C S I}$ is Gaussian, the maximum differential entropy $h\left(\mathbf{d}_{2 I C S I}\right)$ is written as

$$
h\left(\mathbf{d}_{2 I C S I}\right)=\frac{1}{2} \log _{2} 2 \pi e\left|\mathbf{R}_{\mathbf{d}_{2 I C S I} \mid}\right|
$$

where $\mathbf{R}_{\mathbf{d}_{2 / C S I}}$ is the $M \times M$ correlation matrix of the $M \times 1$ received signal vector with ICSI at the destination node and it is defined as

$$
\mathbf{R}_{\mathbf{d}_{2 I C S I}}=E\left[\mathbf{d}_{2 I C S I} \mathbf{d}_{2 I C S I}^{H}\right]
$$

Substituting (2), in (9) it can be expressed as $E\left[\mathbf{d}_{2 I C S I} \mathbf{d}_{2 I C S I}{ }^{H}\right]=E\left[\left(\mathbf{T} \mathbf{\Delta}\left(\hat{\mathbf{w}}_{B L U E}+\mathbf{e}\right)+\mathbf{n}_{d}\right)\left(\mathbf{T} \mathbf{\Delta}\left(\hat{\mathbf{w}}_{B L U E}+\mathbf{e}\right)+\mathbf{n}_{d}\right)^{H}\right]$

Since the $M \times 1$ destination noise vector $\mathbf{n}_{d}, M \times 1$ error vector $\mathbf{e}$ and the estimated $M \times 1$ overall channel vector $\hat{\mathbf{w}}_{B L U E}$ are independent to each other (10), is simplified as

$$
\begin{aligned}
E\left[\mathbf{d}_{2 I C S I} \mathbf{d}_{2 I C S I}{ }^{H}\right]= & E\left[\mathbf{T} \mathbf{\Delta} \hat{\mathbf{w}}_{B L U E} \hat{\mathbf{w}}_{B L U E}{ }^{H} \Delta^{H} \mathbf{T}^{H}\right]+E\left[\mathbf{T} \mathbf{\Delta} \mathbf{e e}^{H} \Delta^{H} \mathbf{T}^{H}\right]+ \\
& E\left[\mathbf{n}_{d} \mathbf{n}_{d}{ }_{d}^{H}\right]
\end{aligned}
$$

Let $\mathbf{R}_{\hat{w}_{B L E}}=E\left[\hat{\mathbf{w}}_{B L U E} \hat{\mathbf{w}}_{B L U E}^{H}\right]$ be the $M \times M$ overall channel covariance matrix and $\mathbf{R}_{\mathbf{n}_{d}}=E\left[\mathbf{n}_{d} \mathbf{n}_{d}^{H}\right]$ be the $M \times M$ noise covariance matrix. Then (11) is rewritten as $E\left[\mathbf{d}_{2 I C S I} \mathbf{d}_{2 I C S I}{ }^{H}\right]=\mathbf{T} \Delta \mathbf{R}_{\hat{w}_{B L E}} \Delta^{H} \mathbf{T}^{H}+\mathbf{T} \boldsymbol{\Delta} \sigma_{\mathbf{e}}^{2} \Delta^{H} \mathbf{T}^{H}+\mathbf{R}_{n_{d}}$

Substituting (9) and (12) in (8), the differential entropy of the $M \times 1$ received signal vector is formulated as

$$
h\left(\mathbf{d}_{2 I C S I}\right)=\frac{1}{2} \log _{2} 2 \pi e\left|\mathbf{T} \Delta \mathbf{R}_{\hat{w}_{B L E}} \Delta^{H} \mathbf{T}^{H}+\mathbf{T} \mathbf{\Delta} \sigma_{\mathrm{e}}^{2} \Delta^{H} \mathbf{T}^{H}+\mathbf{R}_{n_{d}}\right|
$$

The conditional differential entropy $h\left(\mathbf{d}_{2} / \mathbf{s}\right)$ is equal to $h\left(\mathbf{n}_{d}\right)$.Since $\mathbf{n}_{d}$ is Gaussian vector, the conditional differential entropy $h\left(\mathbf{d}_{2} / \mathbf{s}\right)$ is written as

$$
h\left(\mathbf{d}_{2} / \mathbf{s}\right)=h\left(\mathbf{n}_{d}\right)=\frac{1}{2} \log _{2} 2 \pi e\left|\mathbf{T} \Delta \sigma_{\mathbf{e}}^{2} \Delta^{H} \mathbf{T}^{H}+\mathbf{R}_{n_{d}}\right|
$$

By substituting (13) and (14) in (7), the average mutual information of AFWRN with ICSI is given as

$$
\begin{aligned}
I\left(\mathbf{s} ; \mathbf{d}_{2 I C S I}\right)= & \frac{1}{2} \log _{2} 2 \pi e\left|\mathbf{T} \Delta \mathbf{R}_{\hat{w}_{B L E}} \Delta^{H} \mathbf{T}^{H}+\mathbf{T} \boldsymbol{\Delta} \sigma_{\mathbf{e}}^{2} \Delta^{H} \mathbf{T}^{H}+\mathbf{R}_{n_{d}}\right|- \\
& \frac{1}{2} \log _{2} 2 \pi e\left|\mathbf{T} \boldsymbol{\Delta} \sigma_{\mathbf{e}}^{2} \Delta^{H} \mathbf{T}^{H}+\mathbf{R}_{n_{d}}\right|
\end{aligned}
$$

Further, substituting (15) in (6), the capacity is written as

$$
\begin{aligned}
C_{I C S I}= & \frac{1}{2} \log _{2} 2 \pi e\left|\mathbf{T} \mathbf{\Delta} \mathbf{R}_{\hat{w}_{B L U E}} \Delta^{H} \mathbf{T}^{H}+\mathbf{T} \mathbf{\Delta} \sigma_{\mathbf{e}}^{2} \Delta^{H} \mathbf{T}^{H}+\mathbf{R}_{n_{d}}\right|- \\
& \frac{1}{2} \log _{2} 2 \pi e\left|\mathbf{T} \boldsymbol{\Delta} \sigma_{\mathbf{e}}^{2} \Delta^{H} \mathbf{T}^{H}+\mathbf{R}_{n_{d}}\right|
\end{aligned}
$$

Using the properties of determinant $\operatorname{det}(A B)=\operatorname{det}(A) \operatorname{det}(B)$ and $\operatorname{det}(1 / A)=A^{-1}$, the capacity of AFWRN is simplified and written as 
$C_{I C S I}=\frac{1}{2} \log _{2} \mid\left(\mathbf{I}_{M}+\left(\mathbf{T} \boldsymbol{\Delta} \mathbf{R}_{w} \Delta^{H} \mathbf{T}^{H}\left(\mathbf{T} \boldsymbol{\Delta} \sigma_{\mathbf{e}}^{2} \Delta^{H} \mathbf{T}^{H}+\mathbf{R}_{n_{d}}\right)^{-1}\right) \mid\right.$

where, $\mathbf{I}_{M}$ is an $M \times M$ identity matrix. The ergodic capacity of AFWRN system is obtained by computing the statistical averages over the channel realizations. It is given by

$E\left[C_{I C S I}\right\}=\frac{1}{2} E\left[\log _{2} \mid\left(\mathbf{I}_{M}+\left(\mathbf{T} \Delta \mathbf{R}_{w} \Delta^{H} \mathbf{T}^{H}\left(\mathbf{T} \Delta \sigma_{\mathbf{e}}^{2} \Delta^{H} \mathbf{T}^{H}+\mathbf{R}_{n d}\right)^{-1}\right)\right]\right.$

\section{SIMULATION RESULTS}

In this section, the impact of error variance on the ergodic capacity of AFWRN is investigated by simulations. The channel between source node and relay nodes is $g_{i}$, relay nodes and destination nodes $h_{i}$ are assumed to be circularly symmetric Gaussian random variables with unit variances[1] and [12]. The channel estimation error $\mathbf{e}$ and noise sequence are assumed to be circularly symmetric complex Gaussian random variable with zero mean and variance $\sigma_{\mathrm{e}}^{2}$. The SNR is defined as $S N R=\left(P_{S} \times 1\right) / N_{0}=P_{S}$, where $P_{S}$ is the source node power [1] and [12].

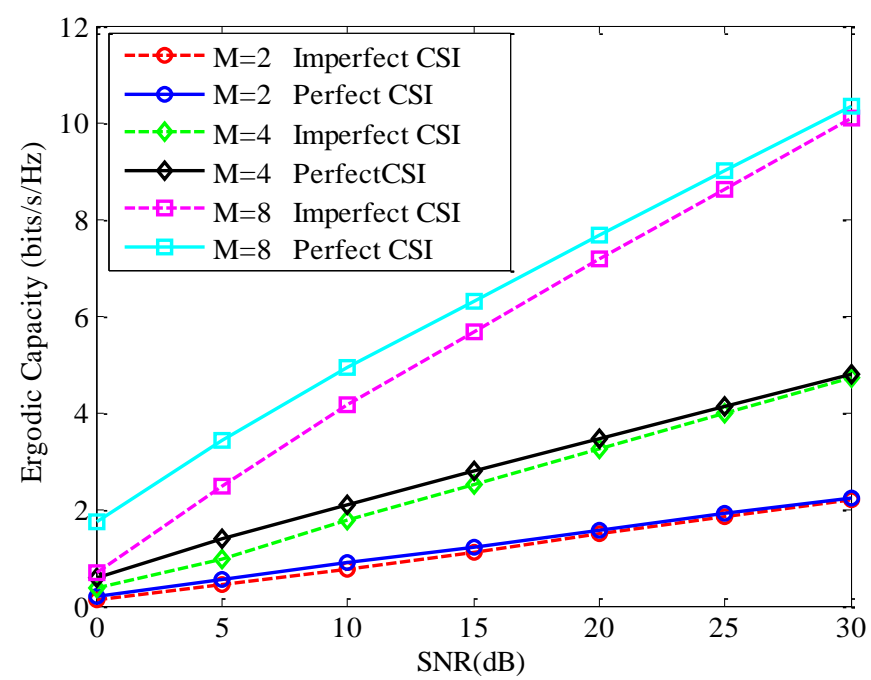

Fig.2 Ergodic Capacity of AFWRN with Perfect CSI and Imperfect CSI

Fig. 2, shows the ergodic capacity of AFWRN by varying the number of relays from $M=2$ to $M=8$ in both Perfect CSI (PCSI) and Imperfect CSI (ICSI) scenarios at the destination node. As the number of relay nodes increases, the ergodic capacity also increases in both PCSI and ICSI scenarios. It is observed that loss between perfect and imperfect scenarios decreases as SNR increases. This is due to the error variance of the BLUE estimator is too small at very high Signal to Noise (SNR). As shown in Fig. 3, the average MSE of the BLUE estimator is $-15 \mathrm{~dB}$ at $10 \mathrm{~dB}$ and $-25 \mathrm{~dB}$ at $20 \mathrm{~dB}$ for the scenario $M=4$ and whereas both LS and MMSE estimator error variances are definitely larger.

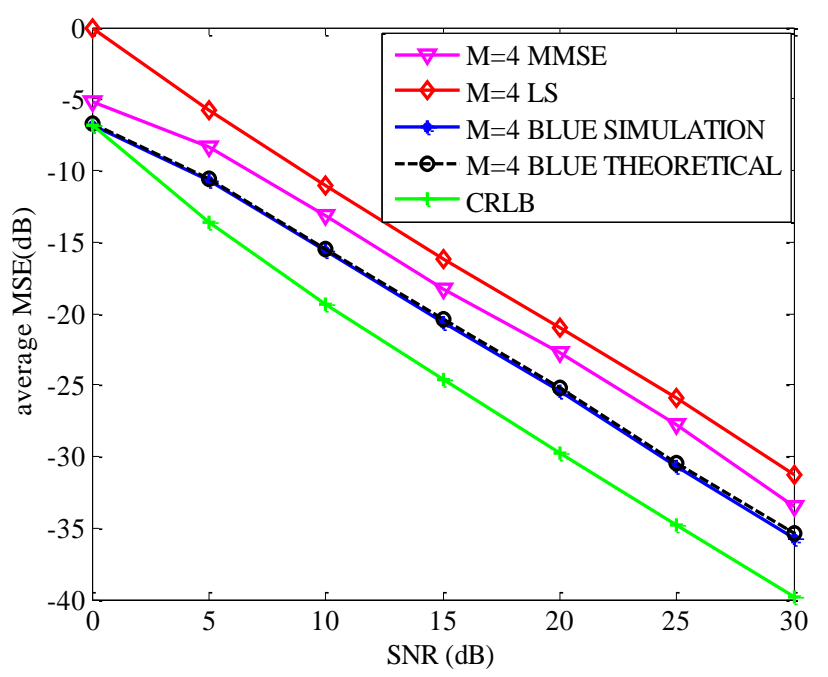

Fig. 3 Average MSE in $\mathrm{dB}$ of Channel Estimation Algorithms at $\mathrm{M}=4$

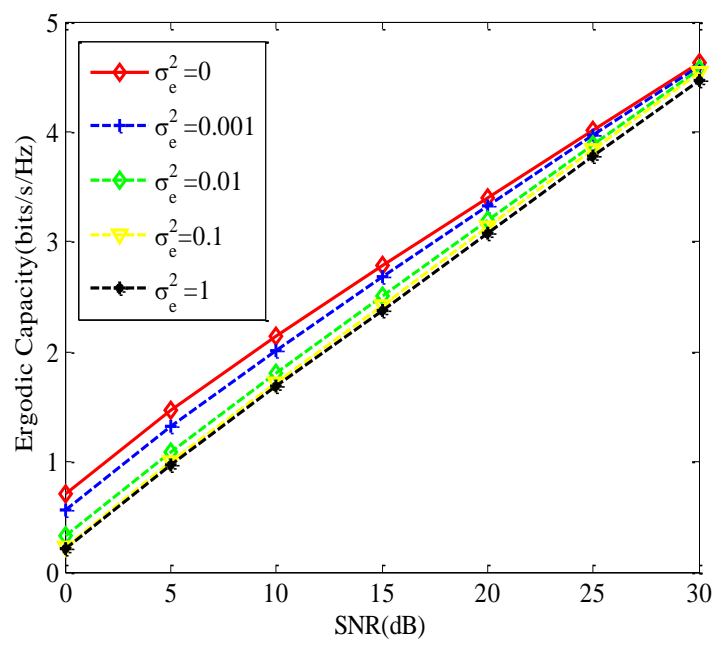

Fig.4 Ergodic Capacity of AFWRN with different values of error variance $\sigma_{\mathbf{e}}^{2}$

Fig.4, shows the ergodic capacity of amplify and forward wireless relay networks for $M=4$ varying SNR 
from 0 to $30 \mathrm{~dB}$ at the error variance of $\sigma_{\mathbf{e}}^{2}=0, \sigma_{\mathbf{e}}^{2}=0.001, \sigma_{\mathbf{e}}^{2}=0.01, \sigma_{\mathbf{e}}^{2}=0.1$ and $\sigma_{\mathbf{e}}^{2}=0.5$. As the error variance of reduces, ergodic capacity increases at high SNR ranges due to increase in SNR and lesser at lower SNR values.Fig. 5, shows ergodic capacity by varying SNR from 0 to $30 \mathrm{~dB}$ with CSI estimated using LS, MMSE and the BLUE algorithm for $M=4$ relay nodes. At $S N R=20 \mathrm{~dB}$, ergodic capacity of the proposed algorithm is $3.4 \mathrm{bits} / \mathrm{s} / \mathrm{Hz}$, whereas the ergodic capacity of LS algorithm and MMSE algorithms are $3.1 \mathrm{bits} / \mathrm{s} / \mathrm{Hz}$ and $3.2 \mathrm{bits} / \mathrm{s} / \mathrm{Hz}$ respectively. The difference in ergodic capacity with LS, MMSE and proposed channel estimation is larger at low SNR regimes. But, the gap between the ergodic capacity with proposed channel estimation algorithm and the perfect CSI is always very minimum.

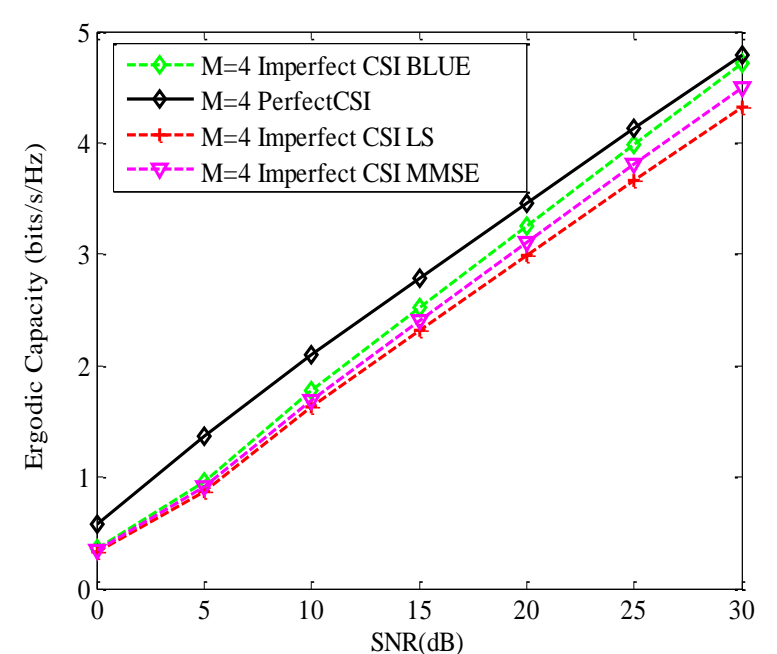

Fig. 5 Ergodic Capacity of AFWRN for various Channel estimation algorithms with PCSI and ICSI

\section{CONCLUSION}

In this paper, ergodic capacity of SISO AFWRN systems with imperfect CSI is analyzed using BLUE algorithm. Due to the minimal variance characteristics offered by BLUE, it shows better performance in comparison to LS and MMSE estimators for ergodic capacity analysis. Hence, this provides a paper provides a new paradigm for ergodic capacity analysis of AFWRN systems and will also afford information theoretic statistics for researchers working in the arena of capacity analysis for wireless networks.

\section{REFERENCES}

[1] F. Gao, T.Cui and A. Nallanathan. "On Channel Estimation and Optimal Training Design for Amplify and Forward Relay Networks", IEEE Trans. Wireless Commun., Vol.7, No.5, pp.1907-1916, May 2008.

[2] O. Dousse M.Franceschetti and P.Thiran "Information Theoretic bounds on the throughput scaling wireless relay networks" in Proc. INFOCOM 2005.

[3] M.A.Khojastepour, A.Sabharwal and B.Azzhang "On the capacity of 'cheap relay networks', in Proc. CISS 2003.

[4] M.Medard "The effect upon channel capacity in wireless communications of Perfect and Imperfect knowledge of the channel", IEEE Trans. Inf. Theory Vol.46, No.3, pp.933-946, May 2000.

[5] A.H.Madsen and Junshan Zhang "Capacity bounds and power allocation for wireless relay channels", IEEE. Trans. Inf. Theory, Vol.51,No.6.pp.2020-2040, June 2003.

[6] G.Farhadi and N.C.Beaulieu "Ergodic Capacity analysis of wireless relaying systems in Rayleigh fading", in Proc.ICC 2008.

[7] Y.Ding, J.K.Zhang and K.M.Wong "Ergodic channel capacity for amplify-forward half-duplex relay system", IEEE Trans.Inf.Theory,Vol.55,No.2,pp.713-730,February 2009.

[8] D.B.da Costa and S. Aïssa "Amplify and forward relaying in channel noise-assisted cooperative networks with relay selection", IEEE Commun.Lett., Vol.14, No.7, pp.608-610, July 2010.

[9] B.Modi, A.Annamalai, O.Olabiyi and C.R.Palat "Ergodic Capacity Analysis of Cooperative Amplify and Forward relay networks over Rice and Nakagami fading channels", Int.J. Wireless Mobile Networks, Vol.4, No.1, pp.97-115, February 2012

[10] Y.Bi and Y.Ding "Relay System at Low SNR in a Generic Noise Environment", IEEE Trans.Signal Process., Vol.60, No.5, pp.2694-2700, May 2012.

[11] A.S.Behbahani and A.Eltawil "On Channel Estimation and Capacity for Amplify and Forward Relay Networks", in Proc. GLOBECOM 2008.

[12] M.PremKumar, V.N.SenthilKumaran and S.J.Thiruvengadam "Channel Estimation Technique for Amplify and Forward Based Wireless Relay Networks using BLUE Approach", in Proc. Wicom 2011. 